\title{
The association of common polymorphisms in the $Q P C T$ gene with bone mineral density in the Chinese population
}

\author{
Qing-Yang Huang · Annie W. C. Kung
}

Received: 25 May 2007 / Accepted: 12 July 2007/Published online: 9 August 2007

(C) The Japan Society of Human Genetics and Springer 2007

\begin{abstract}
Evidence of the linkage of chromosome 2p to bone mineral density (BMD) has previously been reported in multiple populations. However, the identification of the BMD quantitative trait loci (QTL) gene at chromosome $2 p$ remains a challenge. We performed a gene-wide and tag single nucleotide polymorphism (SNP)-based association study of four positional and functional candidate genes (CALM2, CYP1B1, QPCT, and POMC) in a sample of 1,243 cases and matched controls. Thirteen HapMap tag SNPs were selected and genotyped by using the highthroughput Sequenom genotyping platform. Binary logistic regression analyses were performed to test for associations between each SNP genotype and BMD. Haplotype association analyses were performed by WHAP. The rs 3770748 within the $Q P C T$ gene showed a significant association with spine BMD in both singlemarker $(P=0.002)$ and haplotype association analyses ( $P=0.0482$ for the global test; $P=0.00092$ for the haplotype-specific test). Subgroup analysis revealed that the effect was primarily driven by an association in the postmenopausal women, presumably suggesting that the rs3770748 affects postmenopausal bone loss rather than peak bone mass. Our results suggest that QPCT may be the QTL gene at chromosome $2 \mathrm{p}$ for spine BMD variation in the Chinese population.
\end{abstract}

Q.-Y. Huang $(\bowtie) \cdot$ A. W. C. Kung

Department of Medicine, The University of Hong Kong,

Hong Kong, Hong Kong

e-mail: huangqy@hkucc.hku.hk

A. W. C. Kung

e-mail: awckung@hkucc.hku.hk
Keywords Osteoporosis - BMD · Genetics · Association · Tag SNPs

\section{Introduction}

Osteoporosis is characterized by low bone mineral density (BMD) and the development of fractures, particularly of the spine, hip, and wrist. Although the genetic basis of osteoporosis/BMD has been well documented, the genetic causes of osteoporosis remain poorly defined. Genetic linkage analysis and candidate gene association studies have been extensively employed to hunt for osteoporosis susceptibility genes. To date, more than 20 genome-wide linkage scans across multiple populations have been launched (Huang and Kung 2006). Several significant or suggestive chromosomal regions of linkage to BMD have been identified and replicated in genome-wide linkage screens. However, genes that are responsible for these linkage signals remain to be identified.

Several lines of evidence strongly suggest that chromosome $2 p$ is implicated in the regulation of BMD. Devoto et al. (1998) reported a multi-point logarithm of the odds (LOD) score of 2.25 on $2 \mathrm{p} 23$ for spinal BMD in a gemome wide screen of 74 independent sibpairs from 7 large pedigrees. Niu et al. (1999) found linkage evidence of 2p21-23 with forearm BMD with LOD scores of 2.15 in a Chinese population. Kammerer et al. (2003) found significant evidence for quantitative trait loci (QTL) influencing the femoral neck BMD in men on chromosome $2 p$ near D2S1780 (maximum LOD $=3.98$, genomic $P=0.013$ ) in a study of 29 two- and three-generation Mexican-American families using a genome-wide scan. Peacock et al. (2005) identified a QTL for spine BMD with a LOD score of 3.7 on chromosome $2 p$ in 323 pairs of brothers (264 pairs of white 
and 59 pairs of black). Potential functional candidate genes residing on 2p21-23 include the pituitary glutaminyl cyclotransferase $(Q P C T)$, cytochrome P450, subfamily $1 \mathrm{~B}$, polypeptide 1 (CYP1B1), pro-opiomelanocortin (POMC), and calmodulin 2 (CALM2). To identify QTL gene(s) underlying BMD variation at chromosome $2 \mathrm{p}$, we performed a gene-wide and tag single nucleotide polymorphism (SNP)-based association study of the four positional and functional candidate genes in a sample of 1,243 cases and matched controls. Association with SNPs within the QPCT gene was observed.

\section{Materials and methods}

\section{Subjects}

The study subjects came from an expanding database being created at the Osteoporosis Center at Queen Mary Hospital, the University of Hong Kong, Hong Kong, to determine the genetic and environmental risk factors for osteoporosis. All of the study subjects were community-dwelling subjects of southern Chinese descent. These subjects were recruited when they passed by road shows and health talks on osteoporosis held in various districts of Hong Kong between 1998 and 2003. Individuals with disease known to affect bone metabolism, premature menopause (age $<40$ ), bilateral oophorectomy, or drug use that could affect bone turnover and BMD were excluded. All subjects underwent a physical examination and were interviewed by a trained research assistant using a structured questionnaire for their ethnicity, social, medical and reproductive histories, dietary and lifestyle factors, and family history of osteoporosis. A total of 5,872 subjects were invited to the Osteoporosis Center at Queen Mary Hospital for BMD assessment. BMDs $\left(\mathrm{g} / \mathrm{cm}^{2}\right)$ at the L1-4 lumbar spine, femoral neck, trochanter, and total hip were measured by dual-energy $\mathrm{X}$ ray absorptiometry (DXA; Hologic QDR 4500 Plus, Hologic Waltham, MA, USA). The in vivo precision of the machine for the lumbar spine, femoral neck, and total hip regions was $1.2,1.5$, and $1.5 \%$, respectively (Kung et al. 1998). The hip and spine were chosen because they are the most common osteoporotic fracture sites. Weight and height was measured at the same visit when the BMD measurements were taken. To increase the power of the studies, subjects with low and high BMD were identified from these population-based volunteers. A case-control association approach was used, in which the cases were arbitrarily defined as subjects having a low BMD of $Z$ scores $\leq-1.28$ (equivalent to the lowest $10 \%$ of the population) at either the lumbar spine or femoral neck, while the controls were age- and sex-matched subjects with a high $\mathrm{BMD}$ of $Z$ scores $>+1$ at the corresponding sites. We identified 1,243 case-control subjects, with 909 spine casecontrol subjects and 792 femoral neck case-control subjects. Of those, 286 case subjects and 190 control subjects overlapped in both spine and hip analyses. All participants gave informed consent and the study was approved by the Ethics Committee of the University of Hong Kong and conducted according to the Declaration of Helsinki.

\section{Tag SNP selection and genotyping}

Thirteen tag SNPs (tSNP) of the CALM2, CYP1B1, QPCT, and $P O M C$ genes are identified using HapMap (three in $C A L M 2$, three in $C Y P 1 B 1$, five in $Q P C T$, and two in $P O M C$ ) (Table 2). The criterion for tagging was set at $r^{2}>0.8$. SNPs were genotyped by using the high-throughput Sequenom genotyping platform. Briefly, the genotypes were determined with the Homogenous Mass EXTEND assay (Sequenom, San Diego, CA, USA). After polymerase chain reaction (PCR) amplification, nonincorporated dNTPs were removed by shrimp alkaline phosphatase. A detecting primer immediately upstream from the polymorphic site was added, together with a specific combination of deoxy dTTP and di-deoxy dATP, dCTP, dGTP, and thermosequenase (Amersham, Bioscience, Piscataway, NJ, USA). The extension products were then analyzed by mass spectrometry (Sequenom Mass Array System). DNA from case and control subjects were randomly assigned to the 96 well plates, and genotyping was performed blind to the status of the samples. Genotyping was repeated in $5 \%$ of the samples for verification and quality control. In the quality control exercises, the genotype data were confirmed to have an error rate of less than $0.1 \%$.

\section{Statistical analyses}

The genotyping quality of each SNP was first checked for the call rate, minor allele frequency, and Hardy-Weinberg equilibrium. SNPs which did not pass the quality control checks were excluded from further consideration. Binary logistic regression analyses were performed to test for associations between each SNP genotype and BMD, which is implemented in SPSS version 13.0 for Windows (SPSS Inc., Chicago, IL, USA). Haplotype associations were tested by WHAP (http://pngu.mgh.harvard.edu/ purcell/whap/) (Purcell et al. 2007). Haplotype-specific and global tests were performed to assess the association of haplotypes with BMD. Haplotype global tests assess the effects of all haplotypes (if there are $\mathrm{H}$ haplotypes, a single $\mathrm{H}-1$ degrees of freedom test). Haplotype-specific tests evaluate each specific haplotype versus all other haplotypes (i.e., $\mathrm{H} 1$ degrees of freedom tests). 


\section{Results}

The basic characteristics of the study subjects are summarized in Table 1. For the 13 HapMap tSNPs selected, genotyping call rate $>90 \%$, minor allele frequency $>1 \%$, duplicate error rate $<2 \%$, and Hardy-Weinberg equilibrium $P$ values $>1 \%$ were achieved for nine tSNPs. Of these, six tSNPs were mapped within introns and three were in exons. Four tSNPs (rs6713532, rs1693869, rs2302651, and rs2551188) did not pass quality control checks. Of those, three had significant discrepancy with the Hardy-Weinberg equilibrium and one tSNP had genotyping call rates $<90 \%$. Those four tSNPs were not included in further analyses. Details for all tSNPs (including genomic and genic position, minor allele frequency, Hardy-Weinberg equilibrium, and call rate) are shown in Table 2.

Binary logistic regression analyses were performed to test for associations between each SNP genotype and BMD. The results of the single-marker association tests are shown in Table 3. For femoral neck BMD, no significant association was found in our population. For spine BMD, the rs3770748 genotypes showed a significant association with spine BMD $(P=0.002)$. The result remained significant, even after a Bonferroni correction for 18 tests (nine SNPs and two BMD measurements). The genotype frequencies for TT, TC, and CC are $30.7,43.6$, and $25.7 \%$ in control samples, and 26.6, 54.9 , and $18.5 \%$ in case samples. Notably, frequencies of the T/C heterozygotes were significantly higher among case than control subjects $(P=0.001 ; \mathrm{OR}=1.26 ; 95 \% \mathrm{CI}=$ 1.1-1.44). None of the other polymorphisms were associated with spine BMD (Table 4). We further studied the relationship between rs3770748 genotypes and spine BMD in relation to menopausal status. The rs3770748 genotypes were significantly associated with spine BMD in postmenopausal women $(P=0.023)$. The genotype frequencies for TT, TC, and CC are 35.2, 42.9, and $21.9 \%$ in control samples, and $26.6,55.5$, and $17.9 \%$ in case samples. Frequencies of the T/C heterozygotes were significantly higher among case than control subjects $(P=0.006$; OR $=$ $1.3 ; 95 \% \mathrm{CI}=1.07-1.57)$. No association was detected between rs3770748 genotypes and spine BMD in premenopausal women (Table 4).

We further conducted haplotype association analysis across the QPCT gene using WHAP. We identified seven haplotypes, each with the minor allele frequency greater than $3 \%$. Combined, these seven haplotypes accounted for $94.7 \%$ of the chromosomes in this population. We then examined the association of these common haplotypes with spine BMD. The global test of all common haplotype effects revealed a marginally significant association between the $Q P C T$ gene and spine BMD $(P=0.0482)$. Haplotype-specific tests revealed a strong association between the haplotype GCCT and spine BMD $(P=0.00092)$ (Table 5).

\section{Discussion}

Previous studies in different populations have reproducibly shown that chromosome $2 \mathrm{p}$ has a QTL that contributes to BMD variation (Devoto et al. 1998; Niu et al. 1999;
Table 1 Basic characteristics of the 1,243 case-control subjects
Data are expressed as mean $\pm \mathrm{SD}$

\begin{tabular}{|c|c|c|c|c|}
\hline & \multicolumn{2}{|l|}{ Spine } & \multicolumn{2}{|l|}{ Femoral neck } \\
\hline & Case & Control & Case & Control \\
\hline Subject number & 476 & 433 & 427 & 365 \\
\hline Postmenopausal women & 270 & 214 & 235 & 166 \\
\hline Premenopausal women & 137 & 153 & 160 & 162 \\
\hline Men & 69 & 66 & 32 & 37 \\
\hline Age (years) & $53.3 \pm 14.4$ & $53.7 \pm 14.6$ & $51.4 \pm 14.9$ & $51.0 \pm 14.0$ \\
\hline Height (m) & $1.54 \pm 0.08$ & $1.58 \pm 0.07$ & $1.53 \pm 0.08$ & $1.58 \pm 0.07$ \\
\hline Weight (kg) & $49.7 \pm 8.2$ & $63.2 \pm 10.7$ & $48.6 \pm 7.7$ & $64.1 \pm 10.9$ \\
\hline \multicolumn{5}{|l|}{$\operatorname{BMD}\left(\mathrm{g} / \mathrm{cm}^{2}\right)$} \\
\hline Spine & $0.67 \pm 0.10$ & $1.13 \pm 0.11$ & $0.72 \pm 0.14$ & $1.07 \pm 0.13$ \\
\hline Femoral neck & $0.56 \pm 0.09$ & $0.80 \pm 0.13$ & $0.52 \pm 0.08$ & $0.89 \pm 0.10$ \\
\hline Trochanter & $0.47 \pm 0.08$ & $0.71 \pm 0.11$ & $0.46 \pm 0.08$ & $0.75 \pm 0.10$ \\
\hline Total hip & $0.64 \pm 0.10$ & $0.92 \pm 0.13$ & $0.61 \pm 0.10$ & $0.97 \pm 0.11$ \\
\hline \multicolumn{5}{|l|}{$Z$-score } \\
\hline Spine & $-1.99 \pm 0.48$ & $1.73 \pm 0.63$ & $-1.62 \pm 0.79$ & $1.15 \pm 0.94$ \\
\hline Femoral neck & $-1.42 \pm 0.70$ & $0.87 \pm 0.91$ & $-1.80 \pm 0.41$ & $1.60 \pm 0.52$ \\
\hline Trochanter & $-1.44 \pm 0.72$ & $1.03 \pm 0.90$ & $-1.63 \pm 0.64$ & $1.38 \pm 0.83$ \\
\hline Total hip & $-1.61 \pm 0.78$ & $1.01 \pm 0.92$ & $-1.89 \pm 0.63$ & $1.49 \pm 0.78$ \\
\hline
\end{tabular}


Table 2 Tag single nucleotide polymorphism (SNPs) genotyped in case-control association analyses

\begin{tabular}{|c|c|c|c|c|c|c|c|}
\hline Gene & tSNP ID & Genomic position $(b p)^{\mathrm{a}}$ & Genic position & Alleles (major/minor) & MAF & HDW $(P)$ & Call rate \\
\hline \multirow[t]{2}{*}{ POMC } & rs6713532 & 25359368 & Intron 3 & $\mathrm{C} / \mathrm{T}$ & 0.43 & 0.007 & 0.83 \\
\hline & rs934778 & 25363759 & Intron 1 & $\mathrm{~T} / \mathrm{C}$ & 0.11 & 1.00 & 0.93 \\
\hline \multirow[t]{3}{*}{$C A L M 2$} & rs1027478 & 47364560 & Intron 3 & $\mathrm{~A} / \mathrm{G}$ & 0.29 & 0.14 & 0.90 \\
\hline & rs815815 & 47373598 & Intron 2 & $\mathrm{~A} / \mathrm{G}$ & 0.11 & 0.83 & 0.97 \\
\hline & rs1693869 & 47376598 & Intron 2 & $\mathrm{G} / \mathrm{C}$ & 0.05 & 0.007 & 0.88 \\
\hline \multirow[t]{5}{*}{$Q P C T$} & rs2255991 & 37554506 & Exon 3 & G/A & 0.33 & 0.87 & 0.97 \\
\hline & rs2302651 & 37561216 & Intron 3 & $\mathrm{~T} / \mathrm{C}$ & 0.37 & 0.076 & 0.78 \\
\hline & rs2373000 & 37567163 & Intron 4 & $\mathrm{~T} / \mathrm{C}$ & 0.34 & 0.65 & 0.96 \\
\hline & rs3770748 & 37570060 & Intron 5 & $\mathrm{~T} / \mathrm{C}$ & 0.47 & 0.71 & 0.96 \\
\hline & rs2373001 & 37572956 & Intron 6 & $\mathrm{~T} / \mathrm{C}$ & 0.45 & 0.83 & 0.97 \\
\hline \multirow[t]{3}{*}{$C Y P 1 B 1$} & rs1056836 & 38272738 & Exon 4 & $\mathrm{C} / \mathrm{G}$ & 0.10 & 0.053 & 0.97 \\
\hline & rs1056827 & 38276712 & Exon 3 & $\mathrm{G} / \mathrm{T}$ & 0.19 & 0.018 & 0.98 \\
\hline & rs 2551188 & 38277329 & Intron 1 & $\mathrm{C} / \mathrm{T}$ & 0.17 & 0.004 & 0.96 \\
\hline
\end{tabular}

$M A F$ minor allele frequency, $H D W$ Hardy-Weinberg equilibrium

${ }^{a}$ Positions are based on the NCBI web site

Table 3 Results of the single-marker association tests

\begin{tabular}{llll}
\hline Gene & tSNP ID & Spine BMD & Femoral neck BMD \\
\hline POMC & rs934778 & 0.099 & 0.443 \\
CALM2 & rs1027478 & 0.971 & 0.482 \\
& rs815815 & 0.678 & 0.867 \\
QPCT & rs2255991 & 0.815 & 0.807 \\
& rs2373000 & 0.674 & 0.884 \\
& rs3770748 & $\mathbf{0 . 0 0 2}$ & 0.178 \\
& rs2373001 & 0.683 & 0.918 \\
CYP1B1 & rs1056836 & 0.054 & 0.614 \\
& rs1056827 & 0.281 & 0.867 \\
\hline
\end{tabular}

Kammerer et al. 2003; Peacock et al. 2005). One approach to follow up the linkage data is to investigate the association between polymorphisms in the candidate genes within the regions of linkage and BMD. To identify the QTL gene underlying $\mathrm{BMD}$ variation at chromosome $2 \mathrm{p}$, we have targeted four positional and functional candidate genes (CALM2, CYP1B1, QPCT, and POMC), and performed a gene-wide and tSNP-based association study in a sample of 1,243 cases and matched controls. Associations with rs3770748 genotypes within the $Q P C T$ gene for spine BMD were observed in the single-marker analyses $(P=0.002)$ and haplotype association analyses $(P=0.0482$ for the global test; $P=0.00092$ for the haplotype-specific test). Subgroup analysis revealed that the effect was primarily driven by an association in the postmenopausal women, presumably suggesting that the rs3770748 affects postmenopausal bone loss rather than peak bone mass. However, because the genotype frequency of rs3770748 in the premenopausal control subjects is very different from that of postmenopausal controls (Table 4), it is likely that there may be a sampling bias in the premenopausal women, resulting in the failure of detecting significant association. A larger sample size will be needed to confirm the findings in premenopausal women.
Table 4 Case-control genotypic association analysis of rs3770748 and spine bone mineral density (BMD)

\begin{tabular}{|c|c|c|c|c|c|}
\hline \multirow[t]{2}{*}{ Sample } & \multicolumn{3}{|c|}{ Genotypic frequency (\%) } & \multirow[t]{2}{*}{$P$} & \multirow[t]{2}{*}{ Odds ratio (OR) } \\
\hline & TT & $\mathrm{TC}$ & $\mathrm{CC}$ & & \\
\hline \multicolumn{6}{|l|}{ All subjects } \\
\hline Case $(n=455)$ & 26.6 & 54.9 & 18.5 & 0.002 & 1.26 \\
\hline Control $(n=417)$ & 30.7 & 43.6 & 25.7 & & \\
\hline \multicolumn{6}{|c|}{ Postmenopausal women } \\
\hline Case $(n=263)$ & 26.6 & 55.5 & 17.9 & 0.023 & 1.3 \\
\hline Control $(n=210)$ & 35.2 & 42.9 & 21.9 & & \\
\hline \multicolumn{6}{|c|}{ Premenopausal women } \\
\hline Case $(n=129)$ & 25.6 & 52.7 & 21.7 & 0.205 & \\
\hline Control $(n=143)$ & 25.2 & 44.1 & 30.8 & & \\
\hline
\end{tabular}


Table 5 Association analysis of $Q P C T$ gene haplotypes with spine BMD

\begin{tabular}{lll}
\hline Haplotype & Frequency & $P$ \\
\hline Global test & & 0.0482 \\
Specific test & \\
ATTC & 0.276 & 0.604 \\
GTCT & 0.273 & 0.729 \\
GCCT & 0.183 & $\mathbf{0 . 0 0 0 9}$ \\
GCTC & 0.091 & 0.179 \\
GCTT & 0.046 & 0.221 \\
GTTC & 0.044 & 0.398 \\
ATCT & 0.034 & 0.253 \\
\hline
\end{tabular}

Estrogen is essential for skeletal maturation, as well as for the maintenance of bone mass in adulthood, and a lack of estrogen is the main cause of postmenopausal osteoporosis (Riggs and Melton 1986; Armamento-Villareal et al. 1992; Riggs et al. 2002). Hence, factors involved in estrogen homeostasis may affect bone status. The hypothalamic/pituitary gonadal axis controls the serum levels of sex hormones (Herbison and Pape 2001; Kang et al. 2001; Burns and Matzuk 2002). Gonadotropin-releasing hormone is a primary regulator of the hypothalamic/pituitary gonadal axis. The QPCT gene encodes pituitary glutaminyl cyclotransferase that can convert active forms of gonadotropin-releasing hormone peptides to the protected forms by converting the N-terminal glutamine of glutamine peptides into the pyroglutamate, forming a cyclic amide structure by dehydration (Busby et al. 1987; Fisher and Spiess 1987; Schilling et al. 2002). The protective function of the QPCT gene for hypothalamic/pituitary peptide hormones makes it a potential candidate gene. Ezura et al. (2004) previously reported that the QPCT gene affects BMD among postmenopausal women in the Japanese population. In this study, we independently replicated the association between the variant rs3770748 and BMD in the Chinese population. Our results indicated that the $Q P C T$ gene is one of the osteoporosis susceptibility genes.

The rs3770748 is located in intron 5 of the QPCT gene. The association of the rs3770748 heterozygotes with lower BMD is puzzling. The most conventional explanation is that the associations with the rs3770748 heterozygotes might simply reflect linkage disequilibrium with a functional marker, elsewhere in the gene, that affects BMD. A systematic evaluation of other QPCT SNPs throughout coding and noncoding regions of the gene would be required to investigate this. Another possibility is that this is a true example of heterozygote disadvantage (underdominance). Functional analysis of allelic interactions of the $Q P C T$ would be required to investigate this. Underdominance and overdominance are considered to be rare phenomena, but have been observed in model organisms (Peters et al. 2003; Kim et al. 2004; Swanson-Wagner et al. 2006).

The CYP1B1, POMC, and CALM2 genes are also located on chromosome 2p. The CYP450 enzymes metabolize estrogen. The C allele of the CYP1B1 G1294C polymorphism is associated with increased estrogen catabolism and lower BMD (Napoli et al. 2004). POMC, a precursor for adrenocorticotropic hormone, acts on the cortex of the adrenal glands, leading to the production of glucocorticoid. Glucocorticoids diminish calcium absorption and increase renal calcium excretion. A significant association of three variations in the promoter region of the POMC gene (-2353G/A, -2345G/A, and $-2313 \mathrm{~A} / \mathrm{C})$ with radial $\mathrm{BMD}$ was recently observed among adult Japanese women (Sudo et al. 2005). Calmodulin is involved in the differential control of osteoblast proliferation and differentiation (Siddhanti and Quarles 1994). In this study, we also investigated common polymorphisms of these genes, and no significant associations have been found between these gene variants and BMDs at all of the sites examined.

The genome-wide haplotype maps developed by the International HapMap Project provide important resources for the selection of tSNPs in association studies (International HapMap Consortium 2005). Based on detailed information about validated SNPs and haplotype structure, we have chosen 13 tSNPs within four positional and functional candidate genes to use in our association study. Minor allele frequencies of these 13 tSNPs are comparable to those of 45 unrelated Han Chinese people from Beijing. Notably, T allele of the rs3770748 within the QPCT gene has a frequency of 0.522 in Han Chinese in Beijing, 0.398 in Japanese in Tokyo, and 0.9 in Caucasian CEPH samples (Utah residents with ancestry from northern and western Europe). Frequencies of the T/C heterozygotes were 0.6 in Han Chinese in Beijing, 0.614 in Japanese in Tokyo, and 0.167 in Caucasians. Ethnic differentiation of the genetic determination of osteoporosis has previously been observed. For example, the frequencies of the high-fracture-risk allele 's' at the Sp1 site of the collagen IA1 gene were elevated in Caucasians, but absent in East Asian populations (Lambrinoudaki and Kung 2001). Hence, whether the variant rs3770748 within the QPCT gene is associated with spine BMD in Caucasians remains to be documented.

In this study, we did not attempt to systematically genotype the chromosome $2 p$ region; rather, we prioritized four obvious positional and functional candidate genes to test the associations of their gene polymorphisms with BMD variation. Faced with the absence of complete functional information for the majority of genes in this susceptibility locus and limited knowledge of the link between gene function and osteoporosis, we cannot 
exclude other genes in this locus that might contribute to the genetic risk of osteoporosis. This would require highdensity SNP genotyping and comprehensive/unbiased analysis of all candidate genes in this genomic region. However, our results pinpoint $Q P C T$ as a QTL gene at chromosome $2 \mathrm{p}$ for spine BMD variation in an Asian population.

Acknowledgments This project is supported by the Hong Kong Research Grant Council and seed funding for basic research, the University of Hong Kong; the Bone Health Fund, Hong Kong University Foundation; and Matching Grant and the Osteoporosis and Endocrine Research Fund, the University of Hong Kong. QY Huang is partially supported by the KC Wong Education Foundation.

\section{References}

Armamento-Villareal R, Villareal DT, Avioli LV, Civitelli R (1992) Estrogen status and heredity are major determinants of premenopausal bone mass. J Clin Invest 90:2464-2471

Burns KH, Matzuk M (2002) Genetic models for the study of gonadotropin actions. Endocrinology 143:2823-2835

Busby WH, Quackenbush GE, Humm J, Youngblood WW, Kizer JS (1987) An enzyme(s) that converts glutaminyl-peptides into pyroglutamyl-peptides-presence in pituitary, brain, adrenal medulla, and lymphocytes. J Biol Chem 262:8532-8536

Devoto M, Shimoya K, Caminis J, Ott J, Tenenhouse A, Whyte MP, Sereda L, Hall S, Considine E, Williams CJ, Tromp G, Kuivaniemi H, Ala-Kokko L, Prockop DJ, Spotila LD (1998) First-stage autosomal genome screen in extended pedigrees suggests genes predisposing to low bone mineral density on chromosomes 1p, 2p and 4q. Eur J Hum Genet 6:151-157

Ezura Y, Kajita M, Ishida R, Yoshida S, Yoshida H, Suzuki T, Hosoi T, Inoue S, Shiraki M, Orimo H, Emi M (2004) Association of multiple nucleotide variations in the pituitary glutaminyl cyclase gene $(Q P C T)$ with low radial BMD in adult women. J Bone Miner Res 19:1296-1301

Fisher WH, Spiess J (1987) Identification of a mammalian glutaminyl cyclase converting glutaminyl into pyroglutamyl peptides. Proc Natl Acad USA 84:3628-3632

Herbison AE, Pape JR (2001) New evidence for estrogen receptors in gonadotropin-releasing hormone neurons. Front Neuroendocrinol 22:292-308

Huang QY, Kung AWC (2006) Genetics of osteoporosis. Mol Genet Metab 88:295-306

International HapMap Consortium (2005) A haplotype map of the human genome. Nature 437:1299-1320

Kammerer CM, Schneider JL, Cole SA, Hixson JE, Samollow PB, O'Connell JR, Perez R, Dyer TD, Almasy L, Blangero J, Bauer RL, Mitchell BD (2003) Quantitative trait loci on chromosomes $2 p, 4 p$, and $13 q$ influence bone mineral density of the forearm and hip in Mexican Americans. J Bone Miner Res 18:2245-2252
Kang SK, Choi KC, Tai CJ, Auersperg N, Leung PC (2001) Estradiol regulates gonadotropin-releasing hormone $(\mathrm{GnRH})$ and its receptor gene expression and antagonizes the growth inhibitory effects of GnRH in human ovarian surface epithelial and ovarian cancer cells. Endocrinology 142:580-588

Kim KS, Kim JJ, Dekkers JC, Rothschild MF (2004) Polar overdominant inheritance of a DLK1 polymorphism is associated with growth and fatness in pigs. Mamm Genome 15:552-559

Kung AWC, Yeung SSC, Lau KS (1998) Vitamin D receptor gene polymorphisms and peak bone mass in southern Chinese women. Bone 22:389-393

Lambrinoudaki I, Kung AWC (2001) Absence of the high-risk ' $S$ ' allele associated with osteoporosis at the intronic SP1 bindingsite of collagen IA1 gene in Southern Chinese. J Endocrinol Invest 24:499-502

Napoli N, Mumm S, Sheik S, Rini GB, Villareal RC (2004) Effect of CYP450 gene polymorphisms on estrogen metabolism and bone density. J Bone Miner Res 19:S384

Niu T, Chen C, Cordel H, Yang J, Wang B, Wang Z, Fang Z, Schork NJ, Rosen CJ, Xu X (1999) A genome-wide scan for loci linked to forearm bone mineral density. Hum Genet 104:226-233

Peacock M, Koller DL, Fishburn T, Krishnan S, Lai D, Hui SL, Johnston CC, Foroud T, Econs MJ (2005) Sex-specific and nonsex-specific quantitative trait loci contribute to normal variation in bone mineral density in men. J Clin Endocrinol Metab 90:3060-3066

Peters AD, Halligan DL, Whitlock MC, Keightley PD (2003) Dominance and overdominance of mildly deleterious induced mutations for fitness traits in Caenorhabditis elegans. Genetics 165:589-599

Purcell S, Daly MJ, Sham PC (2007) WHAP: haplotype-based association analysis. Bioinformatics 23:255-256

Riggs BL, Melton LJ III (1986) Involutional osteoporosis. N Engl J Med 314:1676-1686

Riggs BL, Khosla S, Melton LJ III (2002) Sex steroids and the construction and conservation of the adult skeleton. Endocr Rev 23:279-302

Schilling S, Hoffmann T, Rosche F, Manhart S, Wasternack C, Demuth HU (2002) Heterologous expression and characterization of human glutaminyl cyclase: evidence for a disulfide bond with importance for catalytic activity. Biochemistry 41:1084910857

Siddhanti SR, Quarles LD (1994) Molecular to pharmacologic control of osteoblast proliferation and differentiation. J Cell Biochem $55: 310-320$

Sudo Y, Ezura Y, Kajita M, Yoshida H, Suzuki T, Hosoi T, Inoue S, Shiraki M, Ito H, Emi M (2005) Association of single nucleotide polymorphisms in the promoter region of the pro-opiomelanocortin gene (POMC) with low bone mineral density in adult women. J Hum Genet 50:235-240

Swanson-Wagner RA, Jia Y, DeCook R, Borsuk LA, Nettleton D, Schnable PS (2006) All possible modes of gene action are observed in a global comparison of gene expression in a maize F1 hybrid and its inbred parents. Proc Natl Acad Sci USA 2:6805-6810 